\title{
Dopaminergic Mechanisms in Actions and Habits
}

\author{
Jeffery R. Wickens, ${ }^{1}$ Jon C. Horvitz, ${ }^{2}$ Rui M. Costa, ${ }^{3}$ and Simon Killcross ${ }^{4}$ \\ ${ }^{1}$ Okinawa Institute of Science and Technology, Okinawa 904-2234, Japan, ${ }^{2}$ Department of Psychology, Boston College, Boston, Massachusetts 02467, \\ ${ }^{3}$ Laboratory for Integrative Neuroscience, National Institute on Alcohol Abuse and Alcoholism, National Institutes of Health, Bethesda, Maryland 20892- \\ 9411, and ${ }^{4}$ School of Psychology, Cardiff University, Cardiff CF10 3AT, United Kingdom
}

Recent studies suggest new ways to interpret dopaminergic actions in goal-directed performance and habitual responding. In the early stages of learning dopamine plays an essential role, but with extended training dopamine appears to play a decreasing role in response expression. Experimental manipulation of dopamine levels alters the correlation of cortical and striatal neural activity in behaving animals, and these dopamine-dependent changes in corticostriatal correlations may be reflected in changes in action selection in the basal ganglia. Consistent with this hypothesis, changes in dopamine signaling brought about by sensitization with amphetamine mimic the transition from goal-directed to habit-based instrumental performance. At the cellular level, dopamine-dependent synaptic plasticity may be important initially, and subsequently lead to more persistent changes that no longer require dopamine. The locus of these actions within the cortical and corticostriatal circuitry is a focus on ongoing research.

Key words: learning; reinforcement; striatum; dopamine; action; habit; cortex; corticostriatal

The contribution of corticobasal ganglia loops to the operation of the cerebral cortex is especially important in goal-directed action, and particularly in making choices among alternative actions. The neostriatum plays a key role in these loops, as the primary recipient of glutamatergic input from nearly all cortical regions, and the recipient of the major dopaminergic input from midbrain dopamine neurons involved in reward processing. Information processing in this circuit is under the control of dopamine-dependent synaptic plasticity in the corticostriatal pathway, and dopaminergic modulation of neostriatal neurons. Although we focus on modulation of corticostriatal loops at the striatal level, dopaminergic projections to the prefrontal cortex may also play an important role.

\section{Dopamine-dependent synaptic plasticity in the corticostriatal pathway}

Dopaminergic modulation of synaptic plasticity in the corticostriatal pathway was suggested as a possible mechanism for learning-related effects of dopamine in the neostriatum some decades ago (Beninger, 1983; Miller, 1988; Wickens and Kotter, 1995) and demonstrated experimentally in brain slices (Wickens et al., 1996). Experimental study of dopamine-dependent synaptic plasticity in the striatum was recently reviewed (Reynolds and Wickens, 2002). Striatal long-term potentiation (LTP) is a dopamine-dependent phenomenon that requires dopamine $D_{1}$ receptor activation (Kerr and Wickens, 2001; Centonze et al., 1999). Using in vivo intracellular recording, Reynolds et al. (2001) showed that LTP can be induced by stimulation of the substantia nigra dopamine cells, using protocols that supported

Received April 13, 2007; revised June 8, 2007; accepted June 8, 2007.

Correspondence should be addressed to Prof. Jeffery R. Wickens, Principal Investigator, Neurobiology Research Unit, Okinawa Institute of Science and Technology, 12-22 Suzaki, Uruma City, Okinawa 904-2234, Japan. E-mail: wickens@oist.jp.

D0I:10.1523/JNEUROSCI.1671-07.2007

Copyright $\odot 2007$ Society for Neuroscience $\quad$ 0270-6474/07/278181-03\$15.00/0 self-stimulation behavior in the same animals. This LTP was blocked in control animals administered a dopamine $\mathrm{D}_{1}$-like receptor antagonist. These findings suggest that stimulation of the substantia nigra may positively reinforce behavior by dopamine $D_{1}$ receptor-dependent potentiation of cortical inputs to the striatum. Consistent with this, recent work has shown dopamine $\mathrm{D}_{1}$ receptor-dependent changes in neural firing patterns in the shell of the nucleus accumbens, related to anticipatory rises in dopamine concentration (Cheer et al., 2007).

Dopamine can cause changes in the coordinated activity of neuronal ensembles in corticostriatal circuits

Dopamine-dependent changes at corticostriatal synapses may have an impact on the temporal coordination of activity in corticobasal ganglia circuits. At the network level, changes in the levels of dopaminergic transmission result in rapid and profound changes in the coordinated activity of neurons in corticostriatal ensembles (Costa et al., 2006). When dopaminergic transmission is high, few neurons in the cortex and striatum display crosscorrelated activity. On the other hand, during states of low dopaminergic transmission, as occur after dopamine depletion, the cross-correlated activity in corticostriatal circuits increases (Raz et al., 2001; Goldberg et al., 2002, 2004; Costa et al., 2006). Consistent with this, low dopaminergic transmission results in a large proportion of cortical and striatal neurons firing preferentially during a particular phase of the local field potential oscillation, whereas during high dopaminergic transmission few neurons fire entrained to the local field potential oscillation (Costa et al., 2006).

These dopamine-dependent changes in corticostriatal synchrony may effect changes in action selection in the basal ganglia. The fact that during high dopaminergic transmission striatal neurons are not entrained to the local field potential implies that whenever an input arrives to the striatum from the cortex, this input will most likely result in an action potential (output), regardless of the phase of the local field potential oscillation at the 
time of the input. This means that, during high dopaminergic transmission, the relationship between input and output is tighter, and does not depend on the local oscillations. The consequence for action selection would be that most inputs received coincidentally with high dopaminergic transmission would be relayed forward. On the other hand, most inputs arriving from the cortex during low dopaminergic transmission, especially those arriving out of phase with the preferred local field potential oscillation, would not necessarily result in an output and, therefore, the correspondence between input and output would be low (i.e., most inputs would be "gated") (Costa, 2007). This suggests that during low dopaminergic transmission it would be difficult to initiate voluntary actions to obtain particular outcomes, whereas during high dopaminergic states it would be difficult to inhibit inappropriate responses. However, training of goal-directed actions can render their performance less dopamine-dependent.

\section{Dopamine $D_{1}$ receptor activation is important for the expression of new appetitive learning}

Expression of simple appetitive responses is dependent on intact dopamine transmission during the early phases of learning, but becomes dopamine-independent with extended training. Rats under conditions of $\mathrm{D}_{1}$ receptor blockade are strongly impaired in generating approach responses to a food compartment in the absence of a salient response-eliciting cue, but can normally initiate the same behavior when it is cued by a well trained conditioned stimulus (CS). After three daily sessions of CS-food pairings (28 trials per session), the $\mathrm{D}_{1}$ antagonist $\mathrm{SCH} 23390$ disrupts both cued and noncued responding. However, after 16 daily sessions, the $\mathrm{D}_{1}$ antagonist (1) continues to reduce the frequency of noncued head entries during the intertrial interval, but (2) produces no impairment in the latency to perform the same head entry behavior in response to the CS (Choi et al., 2005). Because animals receive only a single drug injection, the change in response vulnerability cannot be attributed to repeated drug administration.

The precise neurobiological changes that accompany habit learning remain to be revealed. However, these results suggest two intriguing possibilities. First, dopamine may play a declining role in modulating response expression within dopamineinnervated regions: for example, task-relevant corticostriatal glutamatergic postsynaptic potentials may require amplification by dopamine (Horvitz, 2002) during early stages of learning. During later stages of learning, these glutamatergic synapses may become so efficient that dopamine facilitation of glutamatergic transmission is no longer necessary for normal responding. Alternatively, the behavior may shift with training to representation by nondopamine target areas and, therefore, become less subject to dopamine modulation. It has been suggested that over the course of habit learning, learned sensory-motor representations may shift from corticostriatal-basal ganglia circuits to direct corticocortical mediation (Miller, 1988; Carelli et al., 1997; Ashby et al., 2007).

Increasing dopaminergic activity accelerates habit formation The transition from goal-directed to habit-based instrumental performance is mimicked by sensitization with amphetamine. Nelson and Killcross (2006) demonstrated that repeated injections of amphetamine (seven daily injections, $2 \mathrm{mg} / \mathrm{kg} / \mathrm{d}$ ) in rats 1 week before moderate levels of instrumental lever-press training (three sessions of variable interval training for a total of 120 rewards) enhanced the transition of instrumental control from goal-directed to habitual, indexed by a lack of sensitivity of the instrumental response to reward devaluation by specific satiety prefeeding or with nausea-inducing injections of lithium chlo- ride in a devalued extinction test. Control, vehicle-injected animals and animals receiving sensitization with amphetamine after instrumental training showed normal devaluation of instrumental responding, indicative of goal-directed responding. In all cases, magazine approach responses remained sensitive to devaluation. These results have been extended recently to show that the effect of amphetamine sensitization on habit formation persists for a period of up to 6 weeks after the sensitization injections have ceased, emphasizing the role of chronic pretreatment over acute effects of the psychomotor stimulant, amphetamine (A. Nelson and A. S. Killcross, unpublished observation). In untreated rats, habit-based performance, as assessed by a devaluation procedure, is not normally evident until after considerably more sessions of training (typically 9-10 sessions with some 360400 rewarded lever presses) (Adams, 1982; Killcross and Coutureau, 2003). This finding extends a number of previous reports demonstrating more rapid acquisition of Pavlovian appetitive approach responses after similar sensitization (Harmer and Phillips, 1998), the ability of sensitization with cocaine to render Pavlovian approach responses insensitive to reward devaluation (Schoenbaum and Setlow, 2005), and the effects of overtraining on the sensitivity of Pavlovian approach to dopamine antagonists discussed above (Choi et al., 2005).

Repeated treatment with psychomotor stimulants produces broad-ranging and enduring behavioral consequences, as well as long-term neural adaptations to regions including the striatum (dorsal and ventral), medial prefrontal cortex (Hitchcott et al., 2007), and amygdala (Robinson and Kolb, 2004). Of particular interest in the context of instrumental habit formation are potential effects within the prelimbic and infralimbic regions of the medial prefrontal cortex and the posterior dorsomedial and dorsolateral striatum, all regions shown to be involved in the transition from goal-directed to habitual control of instrumental responding (Coutureau and Killcross, 2003; Yin et al., 2004, 2005; Faure et al., 2005). For example, recent work has demonstrated changes in the network responsiveness of striatal patch and matrix systems (Canales, 2005), with a reduction in the activation of matrix neurons and a recruitment of striosome-based pathways involving limbic prefrontal cortex and basolateral amygdala. Methamphetamine sensitization has been shown to increase spines on medium spiny neurons in the dorsolateral striatum, although reducing them in the dorsomedial subregion (Jedynak et al., 2007), mimicking the potential transition between neural substrates thought to occur in the shift of control from goal-directed to habitual systems brought about by overtraining. Different mechanisms may be involved in medial prefrontal cortex, where direct injections of dopamine have been reported to enhance the role of goal-directed over habitual responding (Hitchcott et al., 2007).

Can cellular and ensemble actions of dopamine explain a shift from goal-directed performance to habitual responding?

During the early stages of learning, when performance is goaldirected, dopamine may play a dual role. In addition to its actions on synaptic plasticity, dopamine modulates the excitability of striatal output neurons by a complex set of actions on voltagedependent and receptor-operated ion channels. The effects of strengthened corticostriatal synapses may be amplified by these actions. Accordingly, during early stages of learning, pharmacological disruptions in dopamine transmission are likely to impair behavioral performance by disrupting the throughput of information across task-relevant corticostriatal synapses. With training, and possibly through persisting changes in synaptic efficacy, the relationship between input and output could become tighter. 


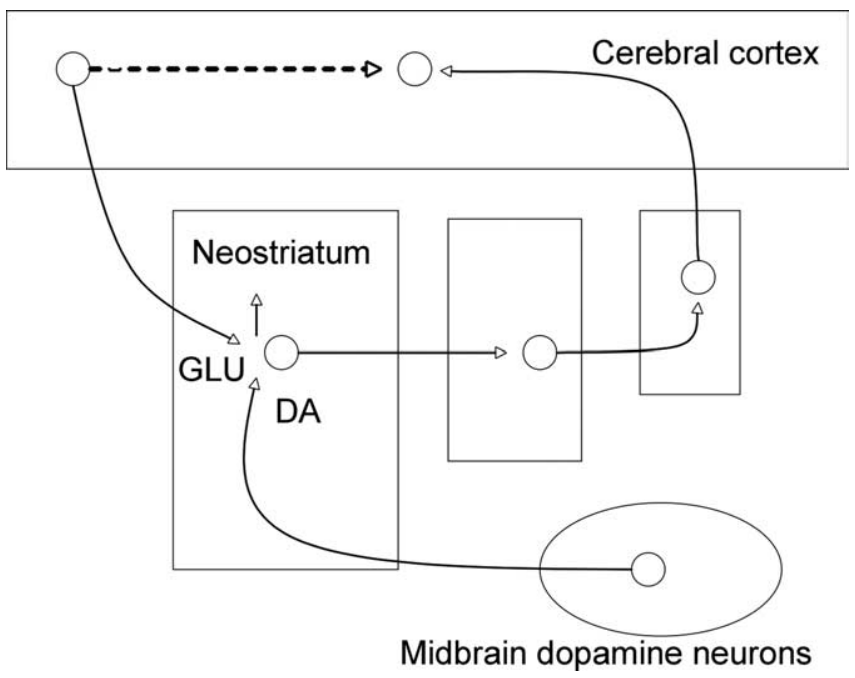

Figure 1. Strengthening corticostriatal connections can lead to increased correlation of cortical and striatal activity, and a shift to $S-R$ responding. The upward arrow indicates that the glutamatergic corticostriatal synapse (GLU) is strengthened by dopaminergic activity (DA), which may also facilitate information transmission by changing the excitability of striatal cells. Connections are shown from the cortex to the striatum and back to cortex via the globus pallidus and thalamus (unlabeled). One possibility would be that DA-mediated strengthening of taskrelevant corticostriatal synapses would eventually render striatal neurons able to fire in the absence of concomitant dopamine release. This strengthening could, with repetition, extend to different striatal subregions than the ones encoding the initial goal-directed behavior (Miyachi et al., 1997; Yin et al., 2004), which would account for the findings that performance of the well established behavior is no longer outcome mediated and less DA dependent. Another possibility would be that direct $S-R$ connections (dotted line) are formed, for example, by the establishment of corticocortical connections (Ashby et al., 2007; Carelli et al., 1997). Neurotransmission via these latter connections would also account for the finding that performance of the well established behavior is no longer outcome mediated.

This may correspond to the apparently decreasing role of dopamine in response expression with extended training. With extended training, operant responses also shift from mediation by outcome value representations to stimulus-response $(\mathrm{S}-\mathrm{R})$ mode performance (Fig. 1). It seems likely that dopaminemediated LTP during learning contributes to the shift to S-R mode performance, because dopamine agonist treatment during learning speeds this shift in processing mode. However, the question of whether the shift to S-R responding and the shift to dopamine-independent response expression coincide remains to be determined.

\section{References}

Adams CD (1982) Variations in the sensitivity of instrumental responding to reinforcer devaluation. Quart J Exper Psychol Comp Physiol Psych 34B:77-98.

Ashby FG, Ennis JM, Spiering BJ (2007) A neurobiological theory of automaticity in perceptual categorization. Psychol Rev, in press.

Beninger RJ (1983) The role of dopamine in locomotor activity and learning. Brain Res: 287:173-196.

Canales JJ (2005) Stimulant-induced adaptations in neostriatal matrix and striosome systems: Transiting from instrumental responding to habitual behavior in drug addiction. Neurobiol Learn Mem 83:93-103.

Carelli RM, Wolske M, West MO (1997) Loss of lever press-related firing of rat striatal forelimb neurons after repeated sessions in a lever pressing task. J Neurosci 17:1804-1814.

Centonze D, Gubellini P, Picconi B, Calabresi P, Giacomini P, Bernardi G (1999) Unilateral dopamine denervation blocks corticostriatal LTP. J Neurophys 82:3575-3579.

Cheer JF, Aragona BJ, Heien ML, Seipel AT, Carelli RM, Wightman RM (2007) Coordinated accumbal dopamine release and neural activity drive goal-directed behavior. Neuron 54:237-244.
Choi WY, Balsam PD, Horvitz JC (2005) Extended habit training reduces dopamine mediation of appetitive response expression. J Neurosci 25:6729-6733.

Costa RM (2007) Plastic corticostriatal circuits for action learning: What's dopamine got to do with it? Ann NY Acad Sci 1104:172-191.

Costa RM, Lin SC, Sotnikova TD, Cyr M, Gainetdinov RR, Caron MG, Nicolelis MA (2006) Rapid alterations in corticostriatal ensemble coordination during acute dopamine-dependent motor dysfunction. Neuron 52:359-369.

Coutureau E, Killcross S (2003) Inactivation of the infralimbic prefrontal cortex reinstates goal-directed responding in overtrained rats. Behav Brain Res 146:167-174.

Faure A, Haberland U, Conde F, El Massioui N (2005) Lesion to the nigrostriatal dopamine system disrupts stimulus-response habit formation. J Neurosci 25:2771-2780.

Goldberg JA, Boraud T, Maraton S, Haber SN, Vaadia E, Bergman H (2002) Enhanced synchrony among primary motor cortex neurons in the 1-methyl-4-phenyl-1,2,3,6-tetrahydropyridine primate model of Parkinson's disease. J Neurosci 22:4639-4653.

Goldberg JA, Rokni U, Boraud T, Vaadia E, Bergman H (2004) Spike synchronization in the cortex/basal-ganglia networks of Parkinsonian primates reflects global dynamics of the local field potentials. J Neurosci 24:6003-6010.

Harmer CJ, Phillips GD (1998) Enhanced appetitive conditioning following repeated pretreatment with d-amphetamine. Behav Pharmacol 9:299-308.

Hitchcott PK, Quinn JJ, Taylor JR (2007) Bidirectional modulation of goaldirected actions by prefrontal cortical dopamine. Cereb Cortex, in press.

Horvitz JC (2002) Dopamine gating of glutamatergic sensorimotor and incentive motivational input signals to the striatum. Behav Brain Res 137:65-74

Jedynak JP, Uslaner JM, Esteban JA, Robinson TE (2007) Methamphetamineinduced structural plasticity in the dorsal striatum. Eur J Neurosci $25: 847-853$

Kerr JN, Wickens JR (2001) Dopamine D-1/D-5 receptor activation is required for long-term potentiation in the rat neostriatum in vitro. J Neurophysiol 85:117-124.

Killcross S, Coutureau E (2003) Coordination of actions and habits in the medial prefrontal cortex of rats. Cereb Cortex 13:400-408.

Miller R (1988) Cortico-striatal and cortico-limbic circuits: a two tiered model of learning and memory function. In: Information processing by the brain: views and hypotheses from a cognitive-physiological perspective (Markowitsch H, ed), pp 179-198. Bern, Switzerland: Hans Huber.

Miyachi S, Hikosaka O, Miyashita K, Karadi Z, Rand MK (1997) Differential roles of monkey striatum in learning of sequential hand movement. Exp Brain Res 115:1-5.

Nelson A, Killcross AS (2006) Amphetamine exposure enhances habit formation. J Neurosci 26:3805-3812.

Raz A, Frechter-Mazar V, Feingold A, Abeles M, Vaadia E, Bergman H (2001) Activity of pallidal and striatal tonically active neurons is correlated in mptp-treated monkeys but not in normal monkeys. J Neurosci 21:RC128.

Reynolds JN, Wickens JR (2002) Dopamine-dependent plasticity of corticostriatal synapses. Neural Netw 15:507-521.

Reynolds JN, Hyland BI, Wickens JR (2001) A cellular mechanism of reward-related learning. Nature 413:67-70.

Robinson TE, Kolb B (2004) Structural plasticity associated with exposure to drugs of abuse. Neuropharmacology 47:33-46.

Schoenbaum G, Setlow B (2005) Cocaine makes actions insensitive to outcomes but not extinction: implications for altered orbitofrontalamygdalar function. Cereb Cortex 15:1162-1169.

Wickens JR, Kotter R (1995) Cellular models of reinforcement. In: Models of information processing in the basal ganglia (Houk JC, Davis JL, Beiser DG, eds), pp 187-214. Cambridge, MA: MIT.

Wickens JR, Begg AJ, Arbuthnott GW (1996) Dopamine reverses the depression of rat cortico-striatal synapses which normally follows high frequency stimulation of cortex in vitro. Neuroscience 70:1-5.

Yin HH, Knowlton BJ, Balleine BW (2004) Lesions of dorsolateral striatum preserve outcome expectancy but disrupt habit formation in instrumental learning. Eur J Neurosci 19:181-189.

Yin HH, Ostlund SB, Knowlton BJ, Balleine BW (2005) The role of the dorsomedial striatum in instrumental conditioning. Eur J Neurosci 22: 513-523. 